Research paper

\title{
Predictive capability of various linearization approaches for floating-ring bearings in nonlinear dynamics of turbochargers
}

\author{
Š. Dyk ${ }^{a, *}$, L. Smolík ${ }^{\mathrm{a}}, \mathrm{J}$. Rendl ${ }^{\mathrm{a}}$ \\ NTIS - New Technologies for the Information Society, Faculty of Applied Sciences, University of West Bohemia, Technická 8, Pilsen, Czech \\ Republic
}

\section{A R T I C L E I N F O}

\section{Article history:}

Received 12 November 2019

Revised 3 February 2020

Accepted 9 February 2020

Available online 2 March 2020

\section{Keywords:}

Floating-ring bearing

Linearization

Dynamic coefficients

Modal analysis

Stability analysis

Turbocharger

\begin{abstract}
A B S T R A C T
A floating-ring bearing (FRB) is composed of a journal, a floating ring and a housing which are separated by two thin oil films. This system is inherently nonlinear and if it is lightly loaded or operated at high speeds, it is prone to the fluid-induced instability. The threshold speed for the instability of a journal bearing with one oil film can be estimated using linearized forces acting in the film. However, the linear analysis of FRB might be problematic because results of such an analysis are usually difficult to interpret. Nevertheless, the linear analysis can provide some fundamental insights into system behaviour. This work aims to evaluate in detail the use of linear theory in the stability analysis of rotating systems supported on FRBs. Several approaches for the linearization of the forces acting in FRB are proposed and analysed. The results are visualized in the form of a holistic Campbell diagram which together depicts natural frequencies, whirl frequencies, modal damping and precession of mode shapes. Moreover, it is demonstrated that the holistic Campbell diagram can be used to interpret - and to some extent, also to predict - results of nonlinear analysis.
\end{abstract}

(c) 2020 Elsevier Ltd. All rights reserved.

\section{Introduction}

Turbochargers are widely used in internal combustion engines due to their ability to improve the engine efficiency. The turbocharger consists of four main components - a bearing housing, two floating ring bearings and a rotor with two overhung impellers. Fig. 1 displays a typical design of the rotor and the bearing system. A thrust bearing, which supports an axial load resulting from aerodynamic forces, is fixed in the bearing housing and radial bearings are located between the turbine and the compressor impellers. Both rolling-element and oil-film bearings with floating rings can be used to support the rotor. The latter is used more often because of a favourable cost and practically unlimited lifetime [1].

However, the floating-ring bearings (FRBs) are subject to the instability of oil films, which can occur in an outer film, in an inner film, or in both films at the same time [2]. Three rotating bodies which spin at various angular speeds - the rotor $\left(\omega_{R}\right)$, the ring at the turbine side $\left(\omega_{F R}^{(L)}\right)$ and the ring at the compressor side $\left(\omega_{F R}^{(R)}\right)$. The inner film is more prone to the instability due to its higher effective hydrodynamic angular velocity. If the inner film loses its stability and oil whirl, which is described e.g. in Ref. [3] occurs, the rotor starts to perform motions at the subsynchronous frequency, which is roughly $0.5\left(\omega_{R}+\omega_{F R}\right)$ [4]. In such a case the stable outer film acts as a damper. Its damping capacity is high enough that the

\footnotetext{
* Corresponding author.

E-mail address: sdyk@ntis.zcu.cz (Š. Dyk).
} 


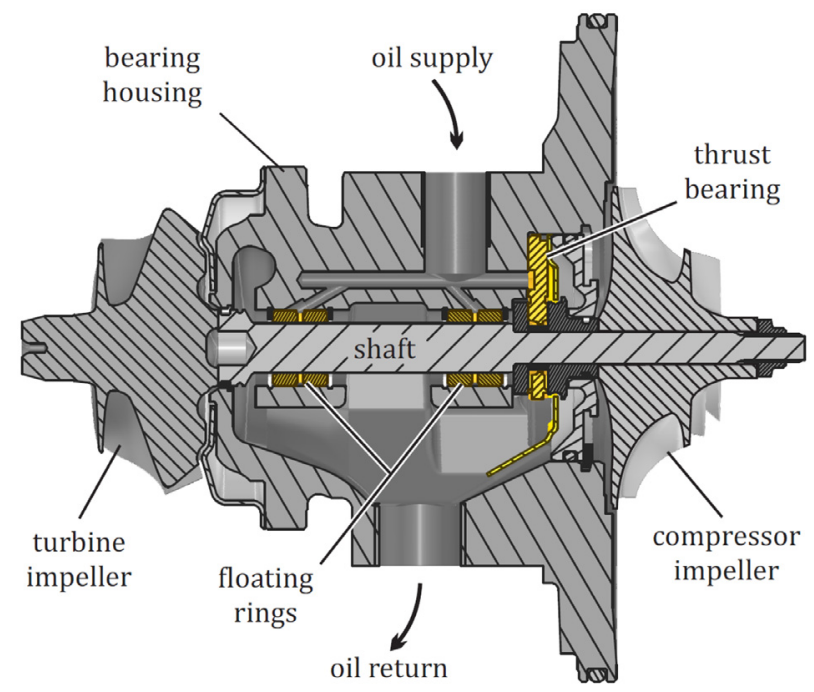

Fig. 1. A typical arrangement of a turbocharger rotor supported on FRBs.

subsynchronous motion reaches a stable limit cycle even if its frequency coincides with a natural frequency of the rotorbearing system [2], i.e. if the oil whirl is replaced by oil whip. When angular speed $\omega_{F R}$ is high enough, the outer film also becomes unstable. The frequency of the whirl of the outer film is approximately $0.5 \omega_{F R}$ and the corresponding whirling motions can appear together with motions due to the instability of the inner film. San Andrés showed that although both motions can exist together, the motion due to the instability of the inner film tends to disappear rather suddenly [4]. This sudden change of the motion can be explained by bifurcation theory and is sometimes referred to as jump (phenomenon) or jumping due to its distinctive appearance in the waterfall plot of vibration spectra [4]. Wang and Khonsari [5] studied bifurcations in rotating systems supported on journal bearings, and Schweizer [2] provided a brief introduction to bifurcations in rotating systems supported on FRBs.

Several distinctive waterfall plots of the turbocharger rotor vibration are reported throughout literature [6-12]. Fig. 2 shows these waterfall plots with removed higher harmonics and sideband frequencies, and a detailed description follows:

(i) The rotor is stable at low speed $\omega_{R}$. A dominant component of rotor's response is a synchronous motion (1X) because of the excitation due to rotating unbalance.

(ii) When rotor speed $\omega_{R}$ increases, the first bifurcation at which the inner film loses its stability is approached. The instability excites a gyroscopic conical deflection shape with forward precession (denoted sub1), and the rotor reaches a stable limit cycle.

Note 1. Response to the rotating unbalance may suppress subsynchronous component sub1, see e.g. [13].

(iii) When rotor speed $\omega_{R}$ increases further, the second bifurcation is reached. There are three possible scenarios:

a) The instability of the inner film starts to excite a gyroscopic cylindrical deflection shape with the forward precession (denoted sub2). The rotor bifurcates from limit cycle (ii) directly into the stable limit cycle consisting of components sub2 and $1 X$, see Fig. 2(a)-(h).

Note 2. In some cases, sub1 is apparent only in a rather narrow interval of rotor speeds and disappears before the second bifurcation is reached. Such a situation is depicted in Fig. $2(\mathrm{~g})$ and $(\mathrm{h})$.

b) Component sub1 disappears and reappears together with sub2 after rotor speed $\omega_{R}$ is sufficiently increased, see Fig. 2(i) and (j).

c) The instability of the outer film starts to excite the gyroscopic conical deflection shape with the forward precession. The rotor bifurcates from limit cycle (ii) directly into the stable limit cycle consisting of components sub3 and $1 X$, see Fig. $2(\mathrm{k})$ and $(\mathrm{l})$.

(iv) If rotor speed $\omega_{R}$ increases even further, additional bifurcations may occur. The number of additional bifurcation points may vary:

a) Component sub2 or sub3 disappears. In such a case, the instability of the oil film is passed through, the rotor becomes stable again and its limit cycle is only constituted of synchronous motion $1 X$. This situation is depicted in Fig. 2(a) and (k).

b) The inner film becomes stable and component sub2 disappears. Further increase of rotor speed $\omega_{R}$ leads to the instability of the outer film and to the introduction of component sub3. The corresponding bifurcation sequence is shown in Fig. 2(b).

Note 3. Direct jumps from sub2 to sub3 are not reported in the reviewed literature. 


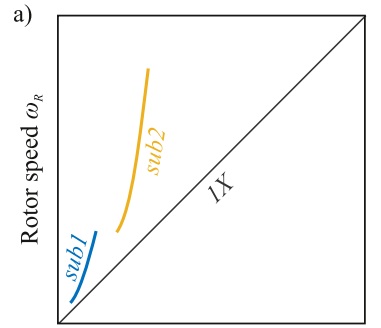

Frequency $f$

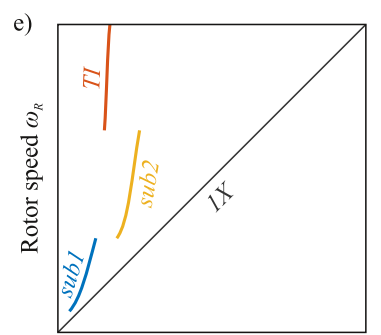

Frequency $f$

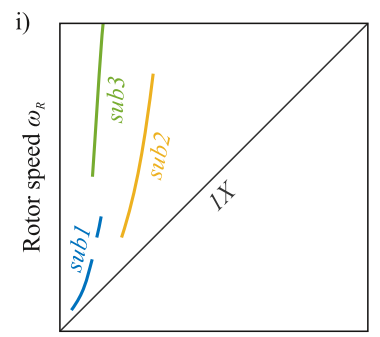

Frequency $f$

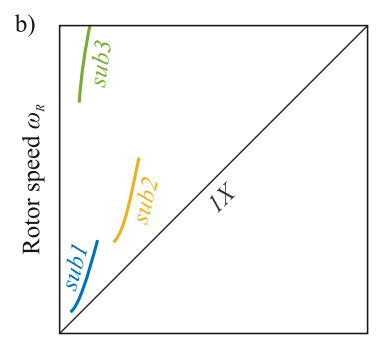

Frequency $f$

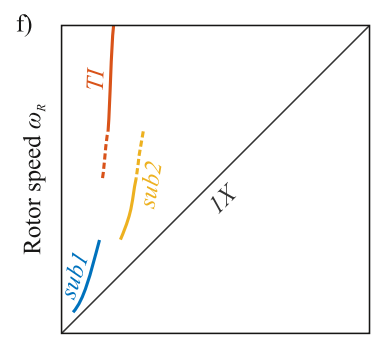

Frequency $f$

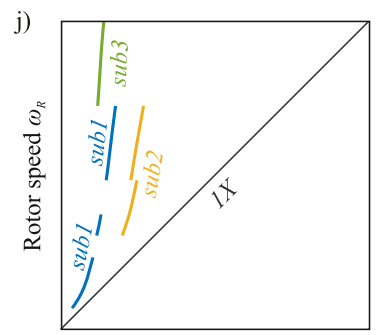

Frequency $f$

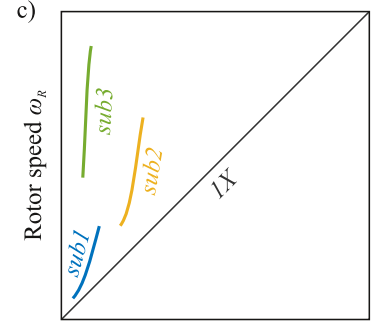

Frequency $f$

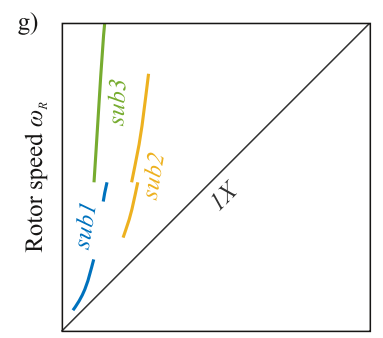

Frequency $f$

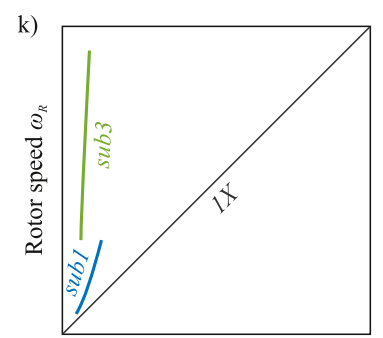

Frequency $f$

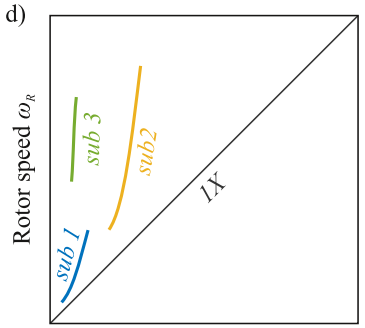

Frequency $f$

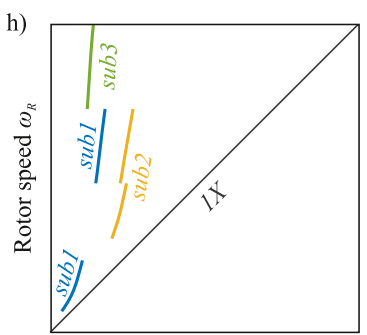

Frequency $f$

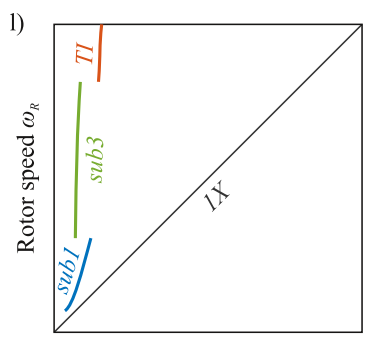

Frequency $f$

Fig. 2. Idealised waterfall plots of the turbocharger rotor vibration with removed higher harmonics and sideband frequencies taken from Refs. [6-12].

c) Additional subsynchronous motion is excited and two subsynchronous components occur simultaneously in rotor's response. The coexistent subsynchronous motions do not meet conditions for an internal resonance phenomenon described e.g. in Ref. [14] and the stable limit cycle can be reached. The bifurcation sequences with coexistent components sub2 and sub3 are depicted in Fig. 2(c), (d), (g), (i), and the sequences with coexistent components sub1 and sub2 are depicted in Fig. 2(g)-(j).

d) Similarly, two subsynchronous components occur simultaneously in rotor's response and they meet conditions for the internal resonance phenomenon. The resulting motion is often called total instability (TI) and prolonged operation under these conditions leads to damage $[2,8]$.

Note 4. Transition from the limit cycle to the total instability may be gradual and a transition zone, which is reported in Ref. [10] and shown in Fig. 2(f), is formed in such a case.

The bifurcation sequences are usually studied employing simulations of the system with nonlinear hydrodynamic forces acting in the bearings. The patterns can be predicted analyzing rotor run-ups [8-12] or a series of steady-state responses $[6,15]$. Tian $[10,13]$ examined the bifurcation sequences acquired from both the run-up and the run-down and found out that they can differ significantly. Schweizer [9] noted that the bifurcation sequence is determined by bearing parameters such as the inner/outer bearing width, the temperature and the pressure of supplied oil, and the outer bearing clearance. Tian $[10,13]$ studied the influence of the outer bearing clearance and the rotor unbalance closely and concluded that both have a major impact on the bifurcation sequences. Smolík et al. [15] later clarified that the stability of the outer film is determined predominantly by the outer clearance. In contrast, the stability of the inner film is more sensitive to the ratio between the inner and outer clearances.

Some authors tried to explain the bifurcation patterns by means of a linear stability analysis: an equilibrium position of the rotor with a given speed is found, the hydrodynamic and gyroscopic forces are linearized and then eigenvalue analysis is performed. Schweizer [9] noted that the first bifurcation occurs due to the loss of stability. Tian [10] showed that up to three unstable eigenvalues can coexist at the given rotor speed. However, the number of the unstable eigenvalues does not indicate how many subsychronous components will be present in the limit cycle at this rotor speed. Woschke [11] proposed a novel method for the construction a holistic Campbell diagram, which is based on the linearization of the hydrodynamic 
forces along the simulated trajectory of the rotor. The resulting Campbell diagram considers changes of the bearing stiffness at the constant rotor speed.

This paper aims to investigate and analyze the possible approaches to the linear modelling of the turbocharger rotors supported on the FRBs thoroughly. The influence of the proposed approaches on modal properties of the rotor-bearing system is discussed, and computational costs of these approaches are examined. Results of the linear analysis are compared with a simulated response of the nonlinear rotor-bearing system, and this comparison is used to illustrate the capability of the linear analysis to characterize the stability of the nonlinear system.

The paper is organized as follows: after the introduction Section 1, mathematical modelling of the rotor supported on the FRBs, linearization process and modal/stability analysis are described in Section 2. Subsequent Section 3 forms a key part of the paper because it describes all the proposed methods. In Section 4, general properties of turbocharger rotors are shown using the Campbell diagrams, computational efficiency is discussed and a comparison of linear analysis with the results of nonlinear run-up simulation is provided. A conclusion follows in Section 5.

\section{Mathematical modeling}

Mathematical background for the modelling of rotors supported on the FRBs is provided in this section. In Section 2.1, a general mathematical model of the rotor is formulated using the finite element method (FEM) for the flexible 1D beam-type continua which is used for the modelling of the shaft. In Section 2.2, detailed pieces of information about the modal analysis and the stability analysis are provided. In the following Section 2.3, linearization of nonlinear bearing forces is commented.

\subsection{Mathematical model of the rotor supported on linearized fluid film bearings}

Mathematical model of the rotor is usually formulated using flexible shaft elements and rigid discs. The shaft can be modelled using the FEM for 1D beam-type continua considering the Euler-Bernoulli, the Timoshenko or other theories.

In the presented paper, the Euler-Bernoulli beam elements are considered with flexural degrees of freedom only (torsional and axial degrees of freedom are neglected due to the assumption of dominant flexural vibration). Vector of generalized coordinates $\boldsymbol{q}_{R}$ of the rotor subsystem rotating at constant speed $\omega_{R}$ is defined in the form

$$
\mathbf{q}_{R}=\left[\ldots, v_{i}, w_{i}, \theta_{i}, \psi_{i}, \ldots\right] \in \mathbb{R}^{n_{R}}, \quad i=1,2, \ldots n_{R}
$$

where $v_{i}, w_{i}$ are the lateral displacements of node $i$ in horizontal and vertical direction, respectively, and $\theta_{i}$, $\psi_{i}$ are the mutually perpendicular bending angles about these displacements. The total number of degrees of freedom is $n_{R}=4 N_{R}$, where $N_{R}$ is the number of nodes.

We assume that shaft deformations are governed by Hooke's law, a rotor subsystem does not have any couplings to the frame or any other subsystems and no external excitation occurs. The equation of motion of such a rotor subsystem can be expressed in a standard matrix form as

$$
\boldsymbol{M}_{R} \ddot{\boldsymbol{q}}_{R}+\left[\boldsymbol{B}_{R}+\boldsymbol{G}_{R}\left(\omega_{R}\right)\right] \dot{\boldsymbol{q}}_{R}+\boldsymbol{K}_{R} \boldsymbol{q}_{R}=\mathbf{0}
$$

where $\boldsymbol{M}_{R}, \boldsymbol{B}_{R}, \boldsymbol{G}_{R}\left(\omega_{R}\right), \boldsymbol{K}_{R} \in \mathbb{R}^{n_{R}, n_{R}}$ are the mass, the damping, the gyroscopic and the stiffness matrices of the rotor, respectively.

In the following definitions of a global model of the turbocharger, two classes of models are distinguished. For the models that neglect floating rings masses equation of motion (2) can be extended by matrices $\boldsymbol{K}_{B}^{(M)}$ and $\boldsymbol{B}_{B}^{(M)}$, which represent stiffness and damping of the bearing coupling, respectively. In such a case no additional degrees of freedom are included and the equation of motion can be rewritten as

$$
\boldsymbol{M}_{R} \ddot{\mathbf{q}}_{R}+\left[\boldsymbol{B}_{R}+\boldsymbol{G}_{R}\left(\omega_{R}\right)+\boldsymbol{B}_{B}^{(M)}\right] \dot{\boldsymbol{q}}_{R}+\left[\boldsymbol{K}_{R}+\boldsymbol{K}_{B}^{(M)}\right] \boldsymbol{q}_{R}=\mathbf{0}
$$

where $M=A, B, C, D$. Generally, coupling matrices $\boldsymbol{K}_{B}^{(M)}, \boldsymbol{B}_{B}^{(M)}$ are a function of shaft speed $\omega_{R}$ for $M=A, B, C$ or a function of the shaft speed $\omega_{R}$ and floating ring speed $\omega_{F R}$ for $M=D$. Particular forms of the coupling matrices are given by the methods for the modelling of the bearing coupling, which are distinguished by indices $M=A, B, C, D$ and which will be dealt with in Section 3.

The second class of models requires the extension of the number of degrees of freedom due to the consideration of floating ring mass. The floating ring is assumed to move only laterally and its angular speed is considered to be constant in the forthcoming analyses. Hence vector of generalized coordinates $\boldsymbol{q}_{F R}^{(X)}$ of the floating ring consists of its horizontal and vertical displacements $v_{F R}^{(X)}$ and $w_{F R}^{(X)}$, respectively, in the form

$$
\boldsymbol{q}_{F R}^{(X)}=\left[v_{F R}^{(X)}, w_{F R}^{(X)}\right]^{T} \in \mathbb{R}^{2}, \quad X=L, R,
$$

where superscripts $L$ and $R$ correspond to the turbine and the compressor bearing, respectively. Then mass matrices of the FRs are given in the form

$$
\boldsymbol{M}_{F R}^{(X)}=\operatorname{diag}\left\{m_{F R}^{(X)}, m_{F R}^{(X)}\right\} \in \mathbb{R}^{2,2}, \quad X=L, R,
$$


where $m_{F R}^{(X)}$ is the mass of the floating ring at the turbine side $(X=L)$ or at the compressor side $(X=R)$. Floating ring rotates at speed $\omega_{F R}^{(X)}$, which can be estimated using the following formula proposed in Ref. [1] under the assumption of zero eccentricity:

$$
R S R_{X}=\frac{\omega_{F R}^{(X)}}{\omega_{R}}=\frac{1}{1+\frac{\mu_{0}}{\mu_{l}} \frac{L_{0}}{L_{l}} \frac{c_{I}}{c_{0}}\left(\frac{D_{0}}{D_{l}}\right)^{3}} \quad X=L, R,
$$

where RSR is the ring speed ratio. Alternatively, RSR can be estimated as a function dependent on relative eccentricity $\varepsilon_{Y}, Y=I, O[1]:$

$$
\operatorname{RSR}_{X}\left(\varepsilon_{I}, \varepsilon_{0}\right)=\frac{\omega_{F R}^{(X)}}{\omega_{R}}=\frac{1}{1+\frac{\mu_{0}}{\mu_{I}} \frac{L_{0}}{L_{I}} \frac{c_{I} \sqrt{1-\varepsilon_{I}^{2}}}{c_{0} \sqrt{1-\varepsilon_{0}^{2}}}\left(\frac{D_{0}}{D_{I}}\right)^{3}} .
$$

Note that (6) and (7) hold under the assumption of a steady-state operation and short bearing approxiamtion with halfSommerfeld cavitation condition.

The equation of motion considering the rotor, two floating rings and linear forces in the bearings can be expressed as

$$
\begin{aligned}
& \operatorname{diag}\left\{\boldsymbol{M}_{R}, \boldsymbol{M}_{F R}^{(L)}, \boldsymbol{M}_{F R}^{(R)}\right\}\left[\begin{array}{c}
\ddot{\boldsymbol{q}}_{R} \\
\ddot{\boldsymbol{q}}_{F R}^{(L)} \\
\ddot{\boldsymbol{q}}_{F R}^{(R)}
\end{array}\right]+\left[\operatorname{diag}\left\{\boldsymbol{B}_{R}+\boldsymbol{G}_{R}\left(\omega_{R}\right), \mathbf{0}, \mathbf{0}\right\}+\boldsymbol{B}_{B}^{(M)}\left(\omega_{R}, \omega_{F R}\right)\right]\left[\begin{array}{c}
\dot{\boldsymbol{q}}_{R} \\
\dot{\boldsymbol{q}}_{F R}^{(L)} \\
\dot{\boldsymbol{q}}_{F R}^{(R)}
\end{array}\right] \\
& +\left[\operatorname{diag}\left\{\boldsymbol{K}_{R}+\boldsymbol{G}_{R}\left(\omega_{R}\right), \mathbf{0}, \mathbf{0}\right\}+\boldsymbol{K}_{B}^{(M)}\left(\omega_{R}, \omega_{F R}\right)\right]\left[\begin{array}{c}
\boldsymbol{q}_{R} \\
\boldsymbol{q}_{F R}^{(L)} \\
\boldsymbol{q}_{F R}^{(R)}
\end{array}\right]=\mathbf{0}
\end{aligned}
$$

which holds for methods $M=E, F, G, I, J$. These methods are described in detail in Section 3.

\subsection{Modal analysis and stability analysis}

Stability of any linear system can be classified based on complex eigenvalues and eigenvectors, which come from solving a modal analysis problem [16-19]. This approach allows to consider a detailed modelling of the investigated rotor system unlike the stability analysis obtained by e.g. Routh-Hourwitz criterion [20] for a simplified 2DoF model of a rigid rotor supported on a journal bearing.

Modal analysis is performed using global mathematical model (3) or (8) with journal bearings described by mass and linearized stifness and damping parameters. The rotor system is not externally excited, see (3) or (8). System of equations (3) or (8) is transformed to state-space for the modal analysis purpose. Association of mass identity $\boldsymbol{M} \dot{\boldsymbol{q}}-\boldsymbol{M} \dot{\boldsymbol{q}}=\mathbf{0}$ to (3) or (8) is utilized. For a shorter notation, $\boldsymbol{M}=\boldsymbol{M}_{R}$ or $\boldsymbol{M}=\operatorname{diag}\left\{\boldsymbol{M}_{R}, \boldsymbol{M}_{F R}^{(L)}, \boldsymbol{M}_{F R}^{(R)}\right\}$ is the global mass matrix for the corresponding approach to the modelling of the bearing coupling and $\dot{\boldsymbol{q}}=\dot{\boldsymbol{q}}_{R}^{T}$ or $\dot{\boldsymbol{q}}=\left[\dot{\boldsymbol{q}}_{R}^{T}, \dot{\boldsymbol{q}}_{F R}^{(L) T}, \dot{\boldsymbol{q}}_{F R}^{(R) T}\right]^{T}$ is the derivative of the vector of generalized coordinates. Thus, eigenvalue problem is generally described for both cases by equation [1]

$$
\left[\lambda_{v}\left(\omega_{R}, \omega_{F R}\right) \boldsymbol{E}-\boldsymbol{A}\left(\omega_{R}, \omega_{F R}\right)\right] \boldsymbol{u}_{v}\left(\omega_{R}, \omega_{F R}\right)=\mathbf{0},
$$

where $\boldsymbol{A}\left(\omega_{R}, \omega_{F R}\right)$ is the system matrix and $\boldsymbol{E}$ is the identity matrix. Eigenvalues are given as complex numbers dependent on the shaft and the floating ring angular speeds:

$$
\lambda_{v}\left(\omega_{R}, \omega_{F R}\right)=\alpha_{v}\left(\omega_{R}, \omega_{F R}\right) \pm \mathrm{i} \beta_{v}\left(\omega_{R}, \omega_{F R}\right) .
$$

Imaginary part $\beta_{\nu}\left(\omega_{R}, \omega_{F R}\right)$ expresses the $v$ th eigenfrequency of the system and the stability is classified based on real part $\alpha_{\nu}\left(\omega_{R}, \omega_{F R}\right)$ of each eigenvalue, called growth/decay rate, see Nguyen-Schäfer [1]. Modal damping factor $D_{\nu}\left(\omega_{R}, \omega_{F R}\right)$ can be used for the stability identification and for a comparison of the magnitude of growth/decay rate at the same time. The modal damping factor is defined as

$$
D_{\nu}\left(\omega_{R}, \omega_{F R}\right)=-\frac{\alpha_{v}\left(\omega_{R}, \omega_{F R}\right)}{\left|\lambda_{v}\left(\omega_{R}, \omega_{F R}\right)\right|} .
$$

The stability can be evaluated using the following rules:

- $\forall v: D_{v} \geq 0$, then the system is stable, and

- $\exists v: D_{v}<0$, then the system is unstable.

Each eigenvalue $\lambda_{v}\left(\omega_{R}, \omega_{F R}\right)$ has corresponding complex eigenvector $\mathbf{u}_{v}\left(\omega_{R}, \omega_{F R}\right)$. Coordinates which correspond with lateral modal displacements can be expressed as

$$
\bar{v}_{j}=v_{j, r}+\mathrm{i} v_{j, i}, \quad \bar{w}_{j}=w_{j, r}+\mathrm{i} w_{j, i},
$$


where $\bar{v}_{j}, \bar{w}_{j}$ are the modal displacements of node $j$ in the horizontal and vertical direction, respectively. After using Euler formula $\mathbf{u}_{v}(t)=\mathbf{u}_{v} \mathrm{e}^{\mathrm{i} \beta_{v} t}$, the lateral modal displacements are described in the time domain as follows

$$
\begin{aligned}
& v_{j}(t)=v_{j, r} \cos \left(\beta_{\nu} t\right)-v_{j, i} \sin \left(\beta_{\nu} t\right), \\
& w_{j}(t)=w_{j, r} \cos \left(\beta_{\nu} t\right)-w_{j, i} \sin \left(\beta_{\nu} t\right) .
\end{aligned}
$$

Hence, the shaft precession angle in the node $j$ is derived as $\psi_{j}=\arctan \left(w_{j}(t) / v_{j}(t)\right)$. The precession direction is then determined from the sign of angle time-derivative $\dot{\psi}_{j}=\mathrm{d} \psi_{j} / \mathrm{d} t$ and supposed shaft rotation direction. After rather lengthy algebraic manipulation, we get final expression [21]

$$
\Psi_{j}=v_{j, i} w_{j, r}-v_{j, r} w_{j, i}
$$

where the sign of indicator $\Psi_{j}$ determines the precession of the motion of node $j$. The precession of the shaft motion can be determined based on the following rules:

- $\forall j: \Psi_{j}>0$, then the shaft whirls with the forward precession,

- $\forall j: \Psi_{j}<0$, then the shaft whirls with the backward precession, and

- $\exists i, j: \Psi_{i}>0 \wedge \Psi_{j}<0$, then the shaft whirls with the combined precession.

\subsection{Linearization of fluid-film forces}

Fluid-film forces generated in thin fluid films are dependent on the pressure distribution which is governed by the Reynolds equation [22]. A general solution of the Reynolds equation in a closed form is not known but Sfyris and Chasalevris [23] found a closed form solution for the homogeneous part of the Reynolds equation and proposed particular solutions in some special cases. Since this analytical solution is rather complicated, several approximate analytical solutions are widely used to approximate fluid film forces. Two most widely known approximate analytical solutions are probably infinitely short (IS) and infinitely long (IL) bearing approximations, which are discussed e.g. in [24]. These two approximate solutions allow a direct expression of the fluid-film forces. Approximate analytical solutions for finite length journal bearings with length-todiameter ratio around 1 also exist $[25,26]$. As a representative of this class of hydrodynamic forces, e.g solution introduced in [24] can be mentioned. This solution is based on the IS approximation but uses multiplicative correction polynomials (further referred to as IScor) to make the solution suitable for the finite length journal bearings.

Above mentioned solution of Reynolds equation and hydrodynamic force must be performed with respect to the chosen boundary cavitation condition [27]. In this paper, the widely used half-Sommerfeld boundary cavitation condition is used. Another boundary cavitation conditions (e.g. Elrod cavitation model) and their mutual comparison in turbocharger applications are introduced in [28].

Even under the above-mentioned simplifying assumptions, the fluid-film forces are naturally nonlinear. However, as the nonlinearities are smooth, the linearization close to the static equilibrium position can be performed.

In principle, linearization process [29-31] is based on the following algorithm ${ }^{1}$ : constant rotor speed $\omega_{R}$ and a constant static bearing load are chosen. Then the static equilibrium position of the journal is found. From the mathematical point of view, the problem is represented by the system of nonlinear algebraic equations. Once the static equilibrium position is determined, the fluid-film forces acting in the bearing are approximated by the Taylor series. Linear terms of the resulting series correspond with stiffness and damping dynamic coefficients. If this process is repeated for other rotor speeds, the dynamic coefficients can be expressed as a function of the rotor speed.

In this article, the following notation will be used for dynamic coefficients (stiffness and damping coefficients, respectively)

$$
k_{i j}^{(X, Y, M)}\left(\omega_{Y}\right), \quad b_{i, j}^{(X, Y, M)}\left(\omega_{Y}\right),
$$

where

- indices $i, j=x, y$ correspond to the horizontal and vertical direction,

- indices $X=L, R$ correspond to the left (compressor) and the right (turbine) bearing,

- indices $Y=I, O$ correspond to the inner $\left(\omega_{I}=\omega_{R}+\omega_{F R}\right)$ and the outer $\left(\omega_{O}=\omega_{F R}\right)$ fluid film, respectively, and

- indices $M=C, D, F, G, I, J$ correspond to the method $^{2}$ chosen for the linearization, see Section 3.

\section{Methods for the description of dynamic coefficients of the FRBs}

In this section, eight possible approaches to the modelling of the FRBs are introduced. The methods are denoted by indices $M=A, \ldots, J$. Methods $M=A, B, C, D$ neglect floating ring degrees of freedom and hence equation of motion (3) holds. For methods $M=E, F, G, I, J$, floating ring mass is considered and extended equation of motion (8) holds.

\footnotetext{
${ }^{1}$ For more precise and detailed formulation, see e.g. [26].

2 Other methods $(M=A, B, E)$ use estimated constant stiffness and damping coefficients and the linearization process is not used in these cases.
} 
Table 1

Overview of the proposed methods.

\begin{tabular}{|c|c|c|c|c|}
\hline Method & Floating ring DoFs & Solution for the equilib. position & RSR calculation & Stiffness and damping coeff. \\
\hline A & none & none & none & isotropic \\
\hline B & none & none & none & orthotropic \\
\hline $\mathrm{C}$ & none & 2 eqs. & eq. (6) & linearized (outer film) \\
\hline $\mathrm{D}$ & none & uncoupled solution ( $2+2$ eqs.) & eq. (6) & linearized (both films) \\
\hline $\mathrm{E}$ & 2 lateral disp. & none & none & isotropic (both films) \\
\hline $\mathrm{F}$ & 2 lateral disp. & uncoupled solution ( $2+2$ eqs.) & eq. (6) & linearized (both films) \\
\hline G & 2 lateral disp. & coupled solution (5 eqs.) & eq. (6) & linearized (both films) \\
\hline I & 2 lateral disp. & coupled solution (5 eqs.) & eq. (7) & linearized (both films) \\
\hline $\mathrm{J}$ & 2 lateral disp. +1 rot. & coupled solution (5 eqs.) & RSR is DoF & linearized (both films) \\
\hline
\end{tabular}

(A)

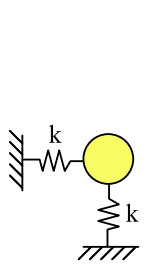

(B)

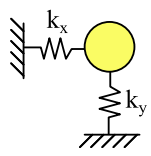

(C)

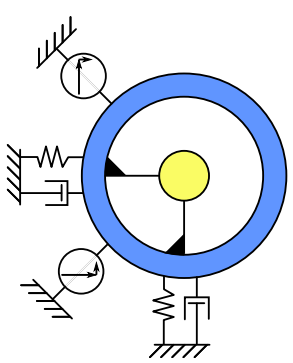

(D)

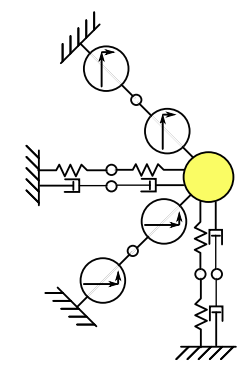

(E)

$(\mathrm{F}, \mathrm{G}, \mathrm{I}, \mathrm{J})$

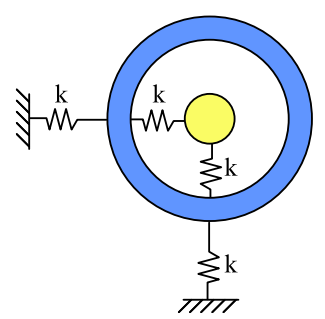

Fig. 3. Schematic diagrams of the bearing couplings using all the considered methods. Cross couplings are denoted by the circles with arrows. Isotropic bearing (A), orthotropic bearing (B), linearized outer fluid film only (C), both films linearized with neglected ring mass (D), both films isotropic considering ring mass and both fluid films linearized with ring mass considered (F,G,I,J). Methods F,G,I,J differ according to the approach to the linearization. Method $\mathrm{F}$ stands for separated linearization for the inner and the outer fluid film, method $G$ means simultaneous linearization for both fluid films, I is the same as $\mathrm{G}$ with different RSR calculation and method J moreover respects angular rotation of floating ring as a degree of freedom.

The description of the methods proceeds from the simplest isotropic (A) and orthotropic (B) bearing approximations, consideration of the linearized outer fluid films only (C) and two linearized dynamic coefficients in series (D) to the models with the floating ring mass and isotropic approximation of both fluid films (E), separated linearization of inner and outer fluid films (F) and coupled linearization (G). The influence of various RSR descriptions is studied using methods (I) and (J). The main assumptions of all the methods are summarized in Table 1.

\subsection{Constant isotropic elastic approximation of bearings $(A)$}

In this first approximation, each FRB can be idealized as two mutually perpendicular linear springs $k \in \mathbb{R}^{+}$, which are shown in Fig. 3(A). Let the nodes in bearings be denoted by indices $i_{L}<i_{R}$ where $i_{X}<N_{R}, X=L, R$. Then coupling matrix $K_{B}^{M}$ from (3) is given as

$$
\boldsymbol{K}_{B}^{(A)}=\operatorname{diag}\{0 \ldots 0, k, k, 0, \ldots 0, k, k, 0 \ldots 0\} \in \mathbb{R}^{n_{R}, n_{R}},
$$

where nonzero elements are localized at the positions corresponding to the horizontal and vertical displacements of nodes $i_{L}, i_{R}$. In this case, stiffness $k$ is assumed to be constant and damping of the bearing is neglected, hence $\boldsymbol{B}_{B}^{(A)}=\mathbf{0} \in \mathbb{R}^{n_{R}, n_{R}}$.

This kind of basic analysis gives an important information about modal properties of the rotor with boundary conditions roughly corresponding to the bearing couplings. However, all of the phenomena affected by the nonlinear nature of the bearings are essentially neglected.

\subsection{Constant orthotropic elastic approximation of bearings (B)}

To demonstrate the influence of different stiffness coefficients in horizontal and vertical direction, orthotropic elastic approximation is considered in this section. Let the stiffness coefficients in horizontal and vertical direction be $k_{x}, k_{y} \in \mathbb{R}^{+}$, respectively. Then, similarly to (17), the coupling stiffness matrix of both bearings can be formulated as

$$
\boldsymbol{K}_{B}^{(B)}=\operatorname{diag}\left\{0 \ldots 0, k_{x}, k_{y}, 0, \ldots 0, k_{x}, k_{y}, 0 \ldots 0\right\} \in \mathbb{R}^{n_{R}, n_{R}}
$$

Both coefficients $k_{x}, k_{y}$ stay constant with the changing rotor speed and the damping is neglected, hence $\boldsymbol{B}_{B}^{(B)}=\mathbf{0} \in \mathbb{R}^{n_{R}, n_{R}}$.

\subsection{Linearization of forces in the outer fluid film (C)}

If the properties of fluid film bearings are taken into account more precisely, linearization process can be used to determine speed dependent stiffness and damping coefficients. 
Due to the fact that the inner clearance of the FRB is usually smaller than the outer clearance, the inner film is stiffer than the outer film and the overall stiffness of the bearing can be approximated by the stiffness of the outer film only. Hence, nonzero elements in the coupling stiffness matrix are given as

$$
{ }^{c} \boldsymbol{K}_{B}^{(X, C)}=\left[\begin{array}{ll}
k_{x x}^{(X, O, C)} & k_{x y}^{(X, O, C)} \\
k_{y x}^{(X, O, C)} & k_{y y}^{(X, O, C)}
\end{array}\right], \quad X=L, R,
$$

where ${ }^{c} \boldsymbol{K}_{B}^{(X, C)}$ is further referred to as the compressed form of the coupling stiffness matrix. The compressed form of coupling damping matrix ${ }^{c} \boldsymbol{B}_{B}^{(X, C)}$ can be derived in a similar manner as ${ }^{c} \boldsymbol{K}_{B}^{(X, C)}$. Then full coupling matrices $\boldsymbol{K}_{B}^{(C)}=$ $\boldsymbol{K}_{B}^{(C)}\left(\omega_{R}\right), \boldsymbol{B}_{B}^{(C)}=\boldsymbol{B}_{B}^{(C)}\left(\omega_{R}\right)$ are zero matrices with only nonzero elements at positions corresponding to the position of horizontal and vertical displacements of nodes $i_{R}, i_{L}$ in the form

$$
\boldsymbol{K}_{B}^{(C)}=\sum_{X=L, R} \boldsymbol{K}_{B}^{(X, C)} .
$$

\subsection{Linearization of forces in both films with neglect of the floating ring mass (D)}

More precisely, both inner and outer fluid films can be considered while the ring mass is still neglected. In this case, linearized dynamic coefficients of the inner and the outer fluid films can be considered to be two springs (or dampers) in series, both speed dependent. The compressed form of the coupling stiffness matrix is

$$
{ }^{c} \boldsymbol{K}_{B}^{(X, D)}=\left[\begin{array}{ll}
k_{x x}^{(X, D)} & k_{x y}^{(X, D)} \\
k_{y x}^{(X, D)} & k_{y y}^{(X, D)}
\end{array}\right], \quad X=L, R,
$$

where

$$
\frac{1}{k_{i j}^{(X, D)}}=\frac{1}{k_{i j}^{(X, I, D)}}+\frac{1}{k_{i j}^{(X, O, D)}} .
$$

Analogical relations also hold for the coupling damping matrix. The localization of nonzero elements in the global coupling matrices and their summation is performed as in previous method C.

\subsection{Constant isotropic elastic approximation of bearings considering the floating ring mass (E)}

Next, floating rings masses and the constant isotropic stiffness approximation of both inner and outer fluid films can be considered. Then equation of motion (8) holds. The compressed form of the coupling stiffness matrix for both fluid films is given as follows

$$
{ }^{c} \boldsymbol{K}_{B}^{(X, E)}=\left[\begin{array}{cccc}
k & & -k & \\
& k & & -k \\
-k & & 2 k & \\
& -k & & 2 k
\end{array}\right], \quad X=L, R .
$$

Nonzero blocks are located at the positions corresponding to the horizontal and the vertical displacements of nodes $i_{L}$, $i_{R}$ and the appropriate floating rings displacements. In this case, damping is neglected $\left(\boldsymbol{B}_{B}^{(E)}=\mathbf{0}\right)$ and after the localization into global stiffness matrix, global coupling matrix of the FRBs can be written in the form

$$
\boldsymbol{K}_{B}^{(E)}=\sum_{X=L, R} \boldsymbol{K}_{B}^{(X, E)} .
$$

3.6. Linearization of the forces in the inner and the outer fluid films - separated linearization for both fluid films (F)

For more precise description of the system behaviour, mass of the floating rings can be considered together with the speed dependent dynamic coefficients corresponding to both the inner and the outer fluid films. A basic approach is an independent linearization of the inner and the outer fluid-film forces. For static equilibrium position determination, two simplified systems are considered:

- journal coupled by the inner fluid film with the space-fixed floating ring,

- journal (with added mass of the floating ring) coupled by outer fluid film with housing.

In this way, two pairs of nonlinear algebraic equations are solved separately to find static solution. Nonlinear algebraic equations are set for the inner fluid film as follows

$$
F_{h d, X}^{(X, I)}\left(\omega_{R}+\omega_{F R}\right)=0, \quad F_{h d, y}^{(X, I)}\left(\omega_{R}+\omega_{F R}\right)+F_{0}^{(X)}=0
$$


and similarly for the outer film

$$
F_{h d, X}^{(X, O)}\left(\omega_{F R}\right)=0, \quad F_{h d, y}^{(X, O)}\left(\omega_{F R}\right)+F_{0}^{(X)}-m_{F R}^{(X)} g=0,
$$

where $F_{h d, i}^{(X, Y)}(\omega)$ are the components of the fluid-film force based on IS or IScor models and $F_{0}^{(X)}$ is the vertical load in the bearing node. Presented indices have following notation $i=x, y, Y=I, O, X=L, R$. The floating ring angular speed is calculated employing (6).

Using this approach, the compressed form of the coupling stiffness matrix is in the form

$$
{ }^{c} \boldsymbol{K}_{B}^{(X, F)}=\left[\begin{array}{cccc}
k_{x x}^{(X, I, F)} & k_{x y}^{(X, I, F)} & -k_{x x}^{(X, I, F)} & -k_{x y}^{(X, I, F)} \\
k_{y x}^{(X, I, F)} & k_{y y}^{(X, I, F)} & -k_{y x}^{(X, I, F)} & -k_{y y}^{(X, I, F)} \\
-k_{x x}^{(X, I, F)} & -k_{x y}^{(X, I, F)} & k_{x x}^{(X, I, F)}+k_{x x}^{(X, O, F)} & k_{x y}^{(X, I, F)}+k_{x y}^{(X, O, F)} \\
-k_{y x}^{(X, I, F)} & -k_{y y}^{(X, I, F)} & k_{y x}^{(X, I, F)}+k_{y x}^{(X, O, F)} & k_{y y}^{(X, I, F)}+k_{y y}^{(X, O, F)}
\end{array}\right] .
$$

The localization of these matrices to the global stiffness matrix $\boldsymbol{K}_{B}^{(F)}\left(\omega_{R}\right)$ is given in the similar way as in (24). Analogical terms also hold for damping matrices.

\subsection{Linearization of the forces in the inner and the outer fluid films - coupled linearization for both fluid films $(G)$}

More precisely, the linearization process in the FRB can be performed considering both fluid films at the same time. It means that both subsystems (the journal and the floating ring) can interact, which is not considered in the aforementioned method F. This interaction can be mathematically expressed by a system of five nonlinear algebraic equations, which can be written in the form

$$
\begin{aligned}
& F_{h d, X}^{(X, I)}\left(\omega_{R}+\omega_{F R}\right)=0, \\
& F_{h d, y}^{(X, I)}\left(\omega_{R}+\omega_{F R}\right)+F_{0}^{(X)}=0, \\
& -F_{h d, X}^{(X, I)}\left(\omega_{R}+\omega_{F R}\right)+F_{h d, X}^{(X, O)}\left(\omega_{R}\right)=0, \\
& -F_{h d, y}^{(X, I)}\left(\omega_{R}+\omega_{F R}\right)+F_{h d, y}^{(X, O)}\left(\omega_{R}\right)-m_{F R}^{(X)} g=0, \\
& M^{(X, I)}+M^{(X, O)}=0,
\end{aligned}
$$

where used indices have notation $i=x, y, Y=I, O, X=L, R$. The components of the fluid-film forces $F_{h d, i}^{(X, Y)}(\omega)$ in the inner and the outer fluid films are based on the infinitely short bearing approximation. Static bearing load is designated $F_{0}^{(X)}$. Torque equilibrium between driving and friction torques that act on the inner and the outer surface of the floating ring, respectively, are expressed in (32). Each applied torque consists of two terms and is adopted from [10]. The first term is derived from lubricant friction, see [1], and the second term is caused by a relative offset of the journal and the bearing shell centres. Floating ring speed $\omega_{F R}$ is calculated using (6).

The coupling stiffness and the coupling damping matrices have the compressed forms which correspond with (27) and the global matrices are finally obtained by the same process as given in (24).

3.8. Linearization of forces in the inner and the outer fluid films - coupled linearization for both fluid films with consideration of RSR influence (I)

To make the model more precise, an improvement of the previous models can be achieved if it is considered that the floating ring speed varies with the rotor speed. This approach offers better accuracy because the effective speed of both inner and outer fluid films is dependent on the floating ring speed which is calculated using (7). The floating ring speed is calculated in each step of static solution during the linearization process and it causes effective speed of both fluid films and hence it influences the dynamic coefficients.

\subsection{Linearization of the inner and the outer fluid films - coupled linearization for both fluid films (J)}

Even more precisely, method J performs solution of the static equilibrium position simultaneously for both fluid films considering an angular rotation of the floating ring as an additional degree of freedom. The final system of equations for this coupled solution is formally the same as (28)-(32) but with the major difference that the floating ring angular speed $\omega_{F R}$ is independent on the shaft speed $\omega_{R}$ given by any RSR formulation (6) or (7).

After the linearization of the fluid-film forces in the inner and the outer fluid films, the compressed forms of the coupling stiffness and the coupling damping matrices, which are given by (27), can be localized to the global coupling matrices in the similar way as shown in (24). 


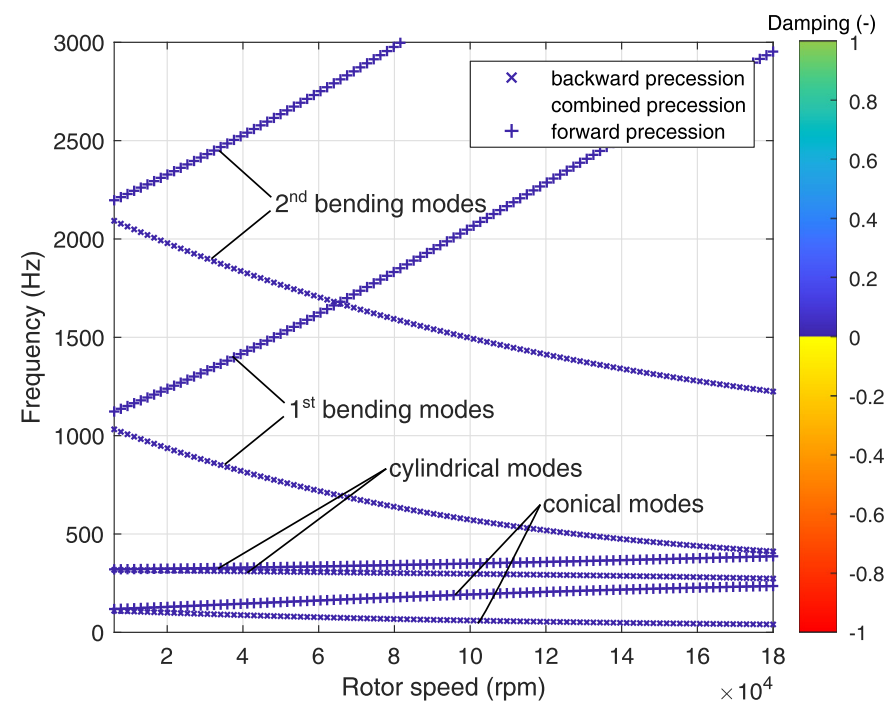

Fig. 4. Campbell diagram for the typical turbocharger considering isotropic linear elastic approximation of the bearings with neglected damping (method A, see Section 3.1). The estimated stiffness of the bearing is $k=10^{6} \mathrm{~N} / \mathrm{m}$.

\subsection{Summary of the proposed methods}

To make a final overview of the proposed methods $M=A, \ldots, J$, a summarizing table is shown in Tab. 1 .

\section{Analysis and results}

In this section, general properties of the turbocharger linear/linearized rotordynamics are shown (Section 4.1), computational demands of the proposed methods are discussed (Section 4.2) and the results of the linear analysis are compared with the results of the nonlinear analysis (Section 4.3).

\subsection{General properties of the linear turbocharger rotordynamics with respect to the approach to the bearing modelling}

In order to investigate varying properties of the modelling approaches presented in Section 3, the presented turbocharger, whose parameters are borrowed from [10], is analyzed. Its parameters are summarised in Table 2. Each of the following Campbell diagrams includes a basic information about the modal properties: basically, it shows speed-dependent eigenfrequencies of the system, the precession of the appropriate mode is indicated by different markers and the modal damping factors are denoted using colours.

A typical Campbell diagram for the turbocharger considering isotropic linear elastic approximation of the bearings (method A) is shown in Fig. 4. The frequency range is limited to $3000 \mathrm{~Hz}$. There are four pairs of branches of natural frequencies there. From the lowest frequency, the pairs are assorted as follows: The backward and forward conical modes (turbine wheel and compressor side in counter-phase) are the first, followed by the backward and the forward cylindrical modes (all the nodes in-phase) and by the backward and the forward first and second bending modes of the shaft.

Method B, see Section 3.2, incorporates orthotropic bearing parameters. In this method it is considered that stiffness $k_{y}$ in the vertical direction is higher than the stiffness $k_{x}$ in horizontal direction, i.e. $k_{y}>k_{x}$. Stiffness parameters are considered $k_{x}=10^{6}[\mathrm{~N} / \mathrm{m}]$ (horizontal stiffness) and $k_{y}=2 k_{x}$. Fig. 5 shows that the quality of the diagram is similar, nevertheless the modes with combined precession appear in some intervals of the rotor speed which has been already presented in Ref. [32].

The Campbell diagram, which is composed employing linearization method $\mathrm{C}$ (the outer film is linearized and the inner film is rigid), is depicted in Fig. 6 . Due to the presence of cross coupling terms $k_{x y}$, $k_{y x}$ and coupling damping matrix, the Campbell diagram becomes more complex. There are unstable branches at frequencies lower than $1000 \mathrm{~Hz}$, which correspond to the conical mode (lower unstable branch) and the cylindrical mode (upper unstable branch). Moreover, it can be seen that the cylindrical mode becomes unstable after crossing a threshold speed.

If both linearized fluid films are taken into account but the ring mass is neglected (method D, see Section 3.4), the model becomes even more complex. Appropriate terms in the coefficient matrices of both fluid films are considered to be speed dependent springs or dampers in series. The corresponding Campbell diagram is shown in Fig. 7. The conical and the cylindrical modes are located at considerably lower frequencies than in the case of the previous method C, see Fig. 6. It is due to the assumption of the rigid inner film. 


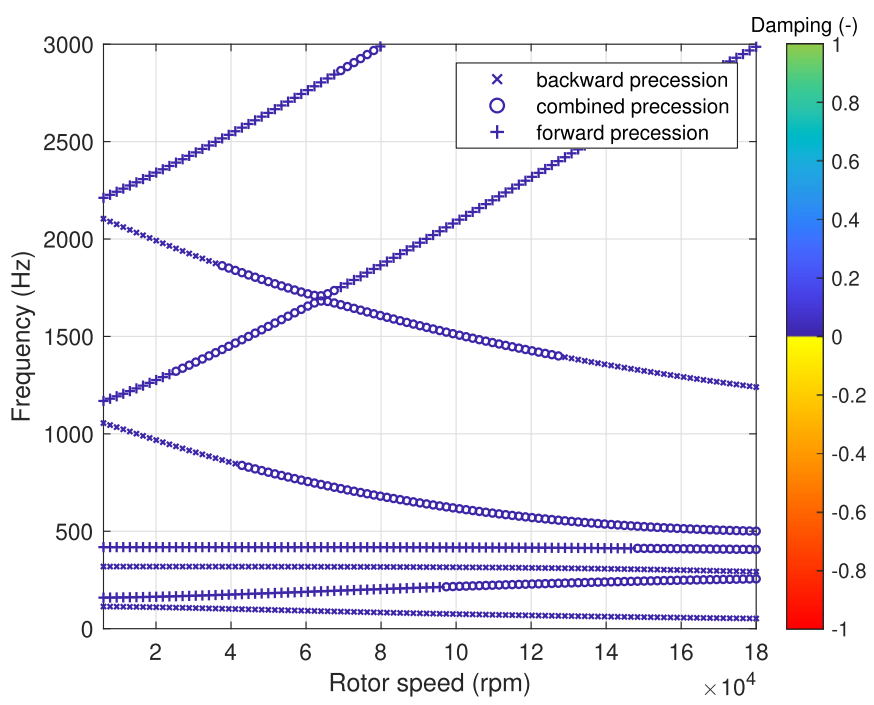

Fig. 5. Campbell diagram for the typical turbocharger considering ortothropic linear elastic approximation of the bearings with neglected damping (method B, see Section 3.2). The stiffness parameters of the bearings are $k_{x}=10^{6} \mathrm{~N} / \mathrm{m}$ (horizontal stiffness) and $k_{y}=2 k_{x}$.

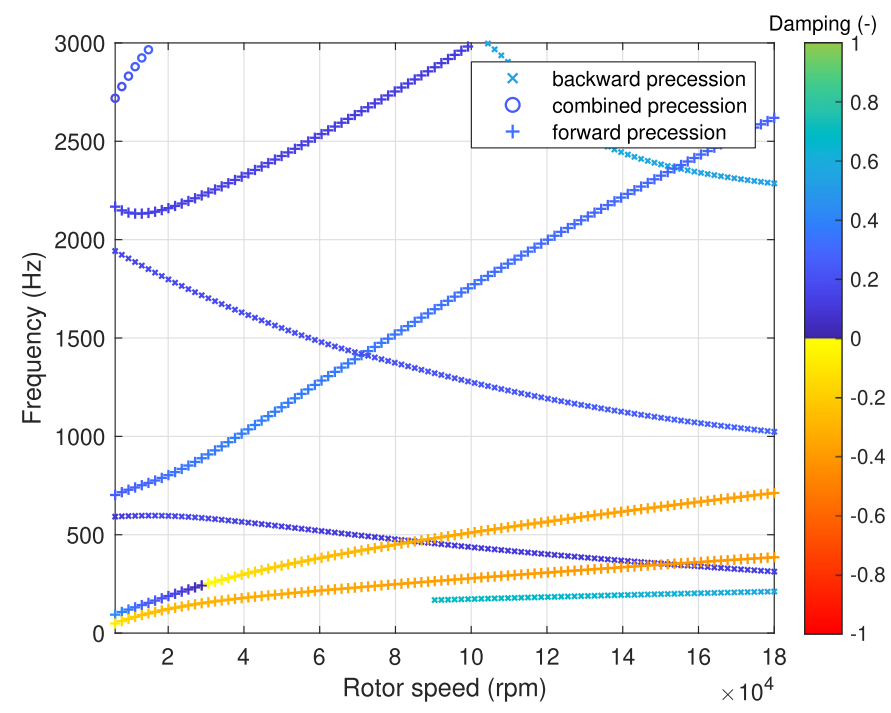

Fig. 6. Campbell diagram for the typical turbocharger (with outer clearance $c_{O}=36 \mu \mathrm{m}$ ) considering linearization of the outer fluid film only (method C, see Section 3.3). Only the modes with damping from range $D \in\langle-1 ; 0.7\rangle$ are depicted for clarity.

The influence of the ring mass is demonstrated in Fig. 8 where the constant isotropic approximation of both fluid films are considered together with the ring mass, see method $\mathrm{E}$ in Section 3.5. The conical and the cylindrical modes are not affected but the second bending modes differ in comparison with the diagrams in Fig. 4. In addition to this difference, two pairs of rings modes appear. These modes are characteristic with large modal displacements of the floating rings and they appear with both backward and forward precession.

From the topological point of view, the most complex linear model includes linearized forces in the inner and the outer films and also takes the ring mass into account. In dependence on the approach used for the linearization (methods F,G,IJ), dynamic coefficients differ, however, the topology of the model is the same in all cases. As an example, Campbell diagram for method $\mathrm{F}$ is shown in Fig. 9. One can observe that there are altogether four branches with unstable conical and cylindrical modes. The mode with the lowest frequency is characterized by the backward precession and it is unstable in the whole rotor speed range. This unstable mode is unlikely to be dangerous for the turbocharger due to its backward precession. Other three branches contain both the stable and the unstable modes with the forward or the combined precession. Interestingly, up to three unstable modes can be found at the single rotor speed. 


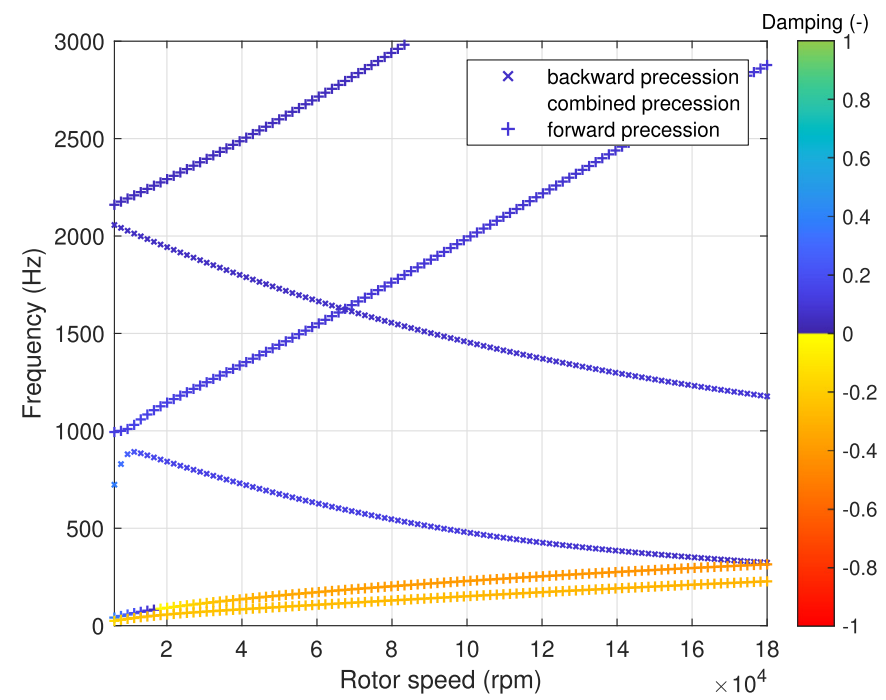

Fig. 7. Campbell diagram for the typical turbocharger (with outer clearance $c_{0}=36 \mu \mathrm{m}$ at both sides) considering linearization of both fluid films and with neglect of the ring mass (method D, see Section 3.3).

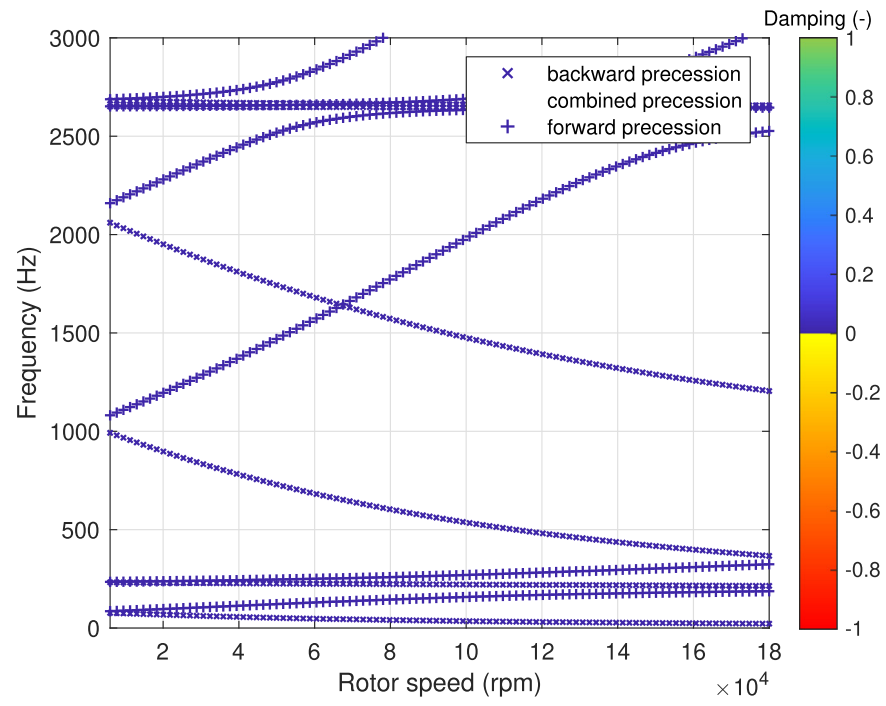

Fig. 8. Campbell diagram for the typical turbocharger considering isotropic linear approximation of the inner and outer fluid films with ring mass; damping is neglected (method E, see Section 3.5).

4.1.1. Comparison of the Campbell diagrams obtained using methods F,G,I,J

If we compare the Campbell diagrams obtained using methods F, G, I and J, only some minor differences in rotor speeds at which individual modes lose or regain the stability can be observed. Eigenfrequencies, precessions and modal damping are affected only slightly.

The estimated floating ring angular speeds expressed by means of the RSR, differ at the methods due to various assumptions. For method F (uncoupled solution) and method G (coupled solution), the RSR is constant and given by (6). The RSR calculation based on (7) is used in method I and floating ring angular speed is supposed to be an additional degree of freedom in method J. A comparison of the RSRs is summarized in Fig. 10. The RSRs used in methods F and G are constant and the same for both bearings because of the identical geometries and oil viscosity. RSR calculation given by (6) is independent on the varying relative eccentricity due to the bearing load. In case of methods I and J, the RSR functions are increasing with higher rotor speeds and the changes tend to be more prominent in the turbine bearing due to a higher static load. As the rotor speed increases, differences among the RSR values obtained by various methods decrease and the RSRs of both rings are becoming more similar. A jump of the RSR function for method $\mathrm{J}$ at low rotor speeds is caused by inaccuracies of the equilibrium position establishment of the system with a free rotating degree of freedom, but the nominal jump discontinuities are negligible. 

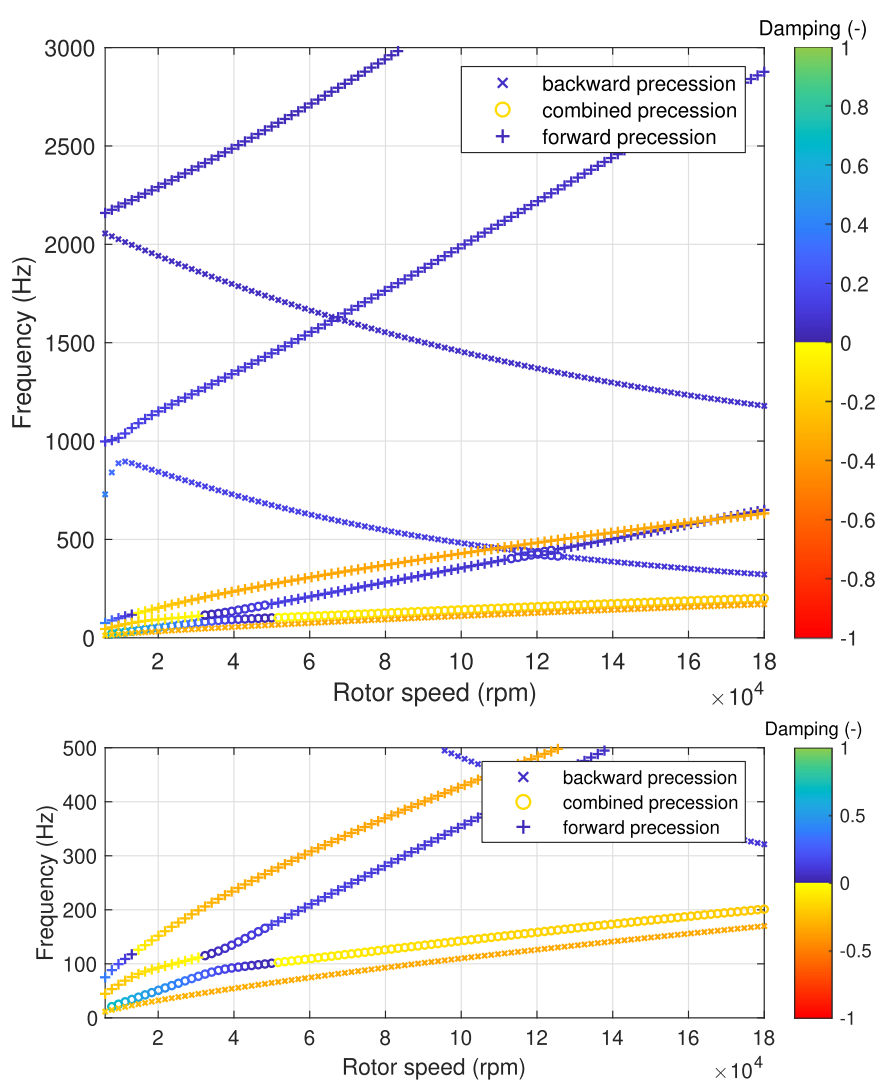

Fig. 9. Campbell diagram for the typical turbocharger considering separated linearization of both fluid films (method F, see Section 3.6). Outer clearance is $c_{0}=36 \mu \mathrm{m}$ at both sides. The lower diagram shows zoom of the upper Campbell diagram in the frequency range from 0 to $500 \mathrm{~Hz}$.
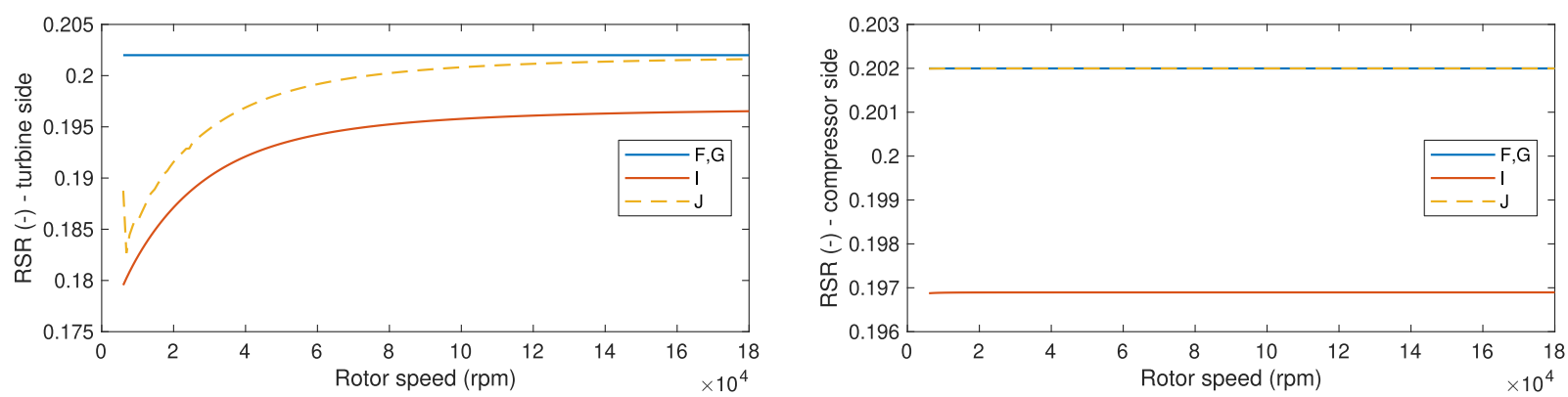

Fig. 10. Mutual comparison of the RSR functions for various methods F, G, I, J.

Although the RSR values vary at the methods, the Campbell diagrams obtained by methods G, I, J do not differ significantly from the diagram for method $\mathrm{F}$, which is depicted in Fig. 9. Hence, making the model more precise (considering a coupled solution, rotation degree of freedom of the floating ring or RSR based on the actual eccentricity during the static solution) does not bring any significant effect to the quality of the Campbell diagram and to the accuracy of the prediction of unstable modes.

\subsection{Computational demands of the proposed methods}

Computational demands of complex modelling methods F, G, I, J are analyzed in this section. In order to compare the computational demands, the CPU time needed for the determination of the static equilibrium is used. Other necessary parts of the computational analysis such as the evaluation of the linearized stiffness and the damping coefficients, modal analysis etc. were omitted because these parts of the computational analysis of the turbocharger do not differ throughout the presented methods or their influence on the total CPU time is negligible. A comparison of computational demands is shown in Fig. 11. The figure consists of the total elapsed time to determine the equilibrium positions and the total number of iterations 

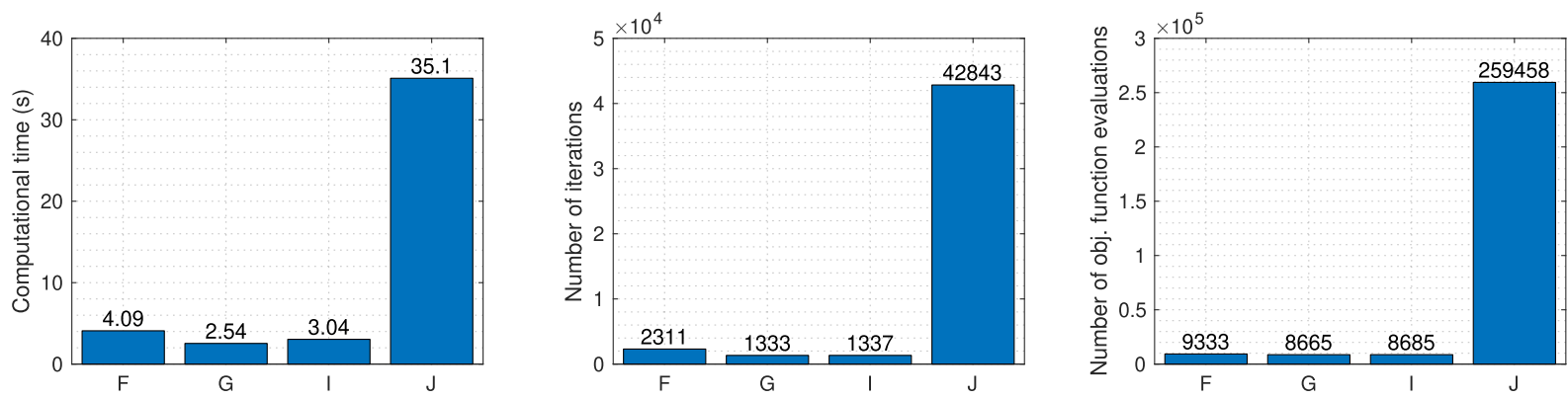

Fig. 11. Computational demands of methods F, G, I, J.

and the objective function evaluation, which were taken during the solution of the system of nonlinear algebraic equations described in Section 3. For the solution of system of equations, the MATLAB built-in function 1sqnonlin was used and the simulations were performed using a regular workstation ${ }^{3}$. Initial conditions for the equilibrium position determination at the specific rotor speed were estimated and for all the investigated methods they were set to be equal.

The highest CPU time is achieved using method J, which assumes free rotation of the floating ring and its independent angular speed, which is calculated in each time-step. The CPU time of method $\mathrm{F}$ is mainly affected by dividing the static equilibrium determination to the separate steps. Obviously, less complicated system of equations is solved twice - for the inner and the outer fluid film. Method G is the least computationally demanding. In contrast to method I, the RSR calculation is based only on the bearing geometry and material properties and does not take into account the position of the journal and the nominal rotor speed. Another efficient method is method I, which has similar demands to method G. However, the results, especially RSR from Fig. 10, are comparable to the more complex method J. Based on the presented study, method I seems to be the best choice for performing the turbochargers linear analysis regarding low demands on the CPU time, good accuracy and complexity of the computational model.

\subsection{Instability thresholds, comparison of the Campbell diagrams with the results of the nonlinear simulations}

In this section, the results of the linearized analysis, which have been shown earlier, will be compared with the runup simulations performed using the full nonlinear model. This model includes nonlinear oil-film forces and no excitation forces such as rotating unbalance force. The simulation is performed in the time-domain using standard ODE solvers for such problems.

Results of both types of the simulations are depicted using composite diagrams. In the case of the linearized analysis, the resulting Campbell diagrams are complemented with curves, which represent whirl frequencies in all four fluid films located in the bearings. Whirl frequency is a theoretical frequency of motion of the journal or the ring in the unstable bearing. The whirl frequencies are calculated analytically in accordance with the theory from Ref. [33] and the onset of instability is evaluated according to the Routh-Hourwitz stability criterion. Time series that represent a response during the run-up is divided into overlapping segments and each segment is analyzed using the fast Fourier transform (FFT). Resulting data are visualised in the form of a spectrogram, which is complemented by dashed and solid curves. The dashed curves are the theoretical whirl frequencies and the solid lines are the frequencies of unstable modes, which are tracked in the Campbell diagram.

The simulations were performed for the outer clearances from range $c_{O}=\langle 36 ; 46\rangle \mu \mathrm{m}$. We assumed that the outer clearances in both bearings $X=L, R$ are equal. As examples, three sets of results are depicted in Figs. 12-14. The results are presented using the composite diagrams described above. The composite Campbell diagrams are based on method J, which is the most complex, because it includes the floating ring mass, the floating ring degree of freedom and coupled linearization of both fluid films in the single bearing. The composite spectrograms show vibrations of both impellers in the range of $25 \mathrm{~dB}$ (2.5 decades). A logarithmic scale with the maximum values presented in yellow and the minimum ones in blue is used. Moreover, the RSR values are shown on the right side of all figures.

The results can be summarized as follows:

- The first bifurcation at which component sub1 appears is predicted rather accurately. The frequency of the motion that is associated with sub1 is also predicted accurately; the accuracy is better at the higher outer clearances.

- The second bifurcation appears only if the mode associated with sub1 is stable. Therefore, if the inner oil film becomes unstable, component sub2 can appear only if the mode associated with component sub1 is stable. Presumably, this is due to the fact that component sub1 is associated with the conical motion, which has lower kinetic energy than the cylindrical motion associated with component sub2.

\footnotetext{
${ }^{3}$ All simulations were performed on an average workstation with processor Intel®Core ${ }^{\mathrm{TM}}$ i7-7700 CPU @ $3.60 \mathrm{GHz}$ (8 CPUs) and 64 GB RAM.
} 

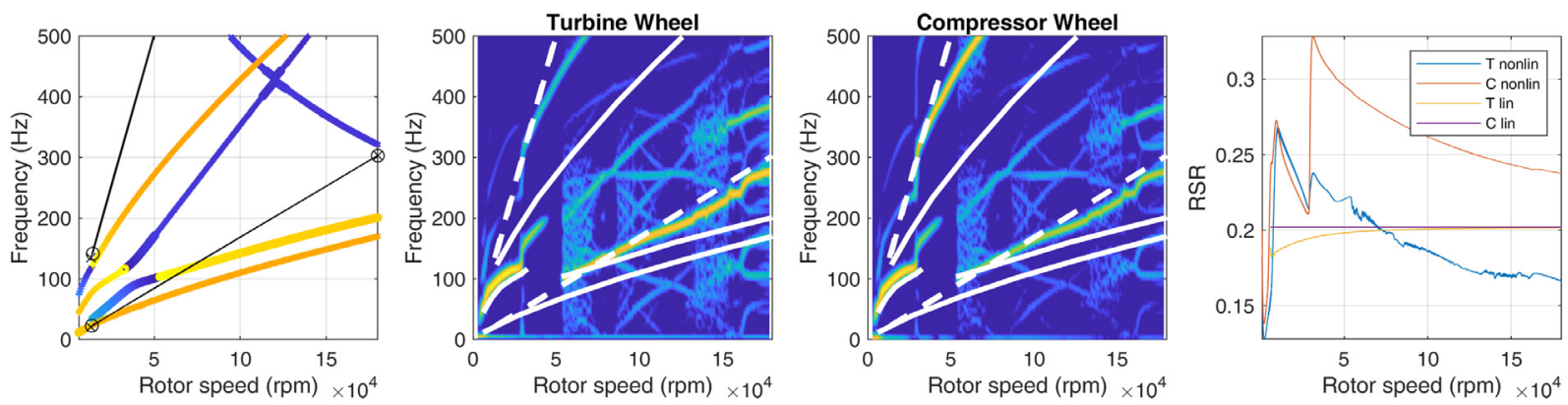

Fig. 12. Comparison of the linearized model $J$ with run-up simulation including nonlinear forces considering outer clearance $c_{0}=36 \mu \mathrm{m}$. Spectrograms depict vibrations of both impellers as a function of the rotor speed, solid white lines correspond to the unstable modes from the Campbell diagram and dashed lines depict the whirl frequency curves. Comparison of the RSRs resulting from the run-up simulation and the linearized model are depicted on the right.
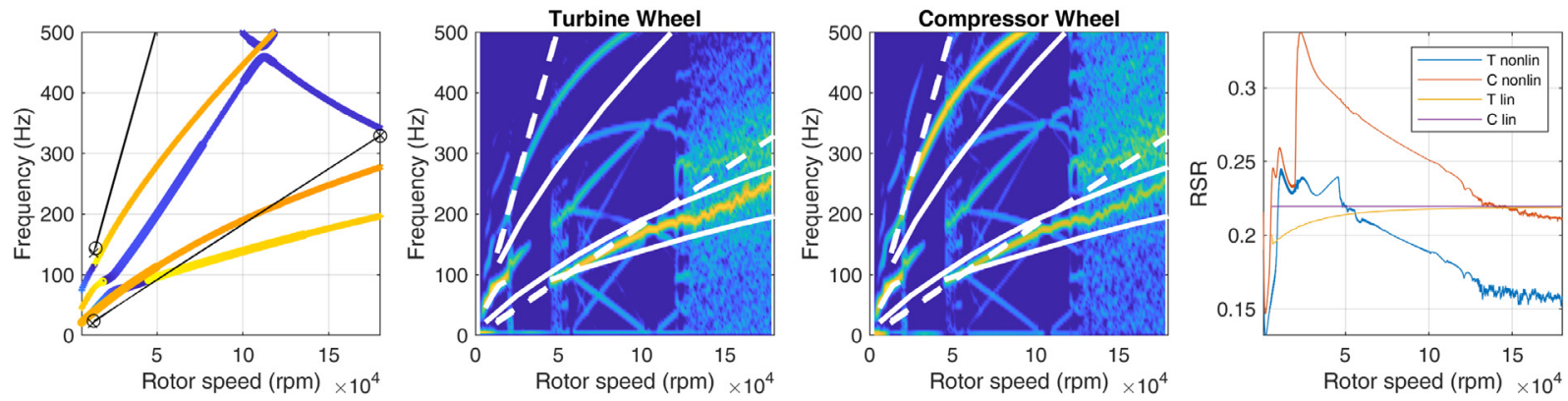

Fig. 13. Comparison of the linearized model $\mathrm{J}$ with run-up simulation including nonlinear forces considering outer clearance $c_{0}=40 \mu \mathrm{m}$. Spectrograms depict vibrations of both impellers as a function of the rotor speed, solid white lines correspond to the unstable modes from the Campbell diagram and dashed lines depict the whirl frequency curves. Comparison of the RSRs resulting from the run-up simulation and the linearized model are depicted on the right.
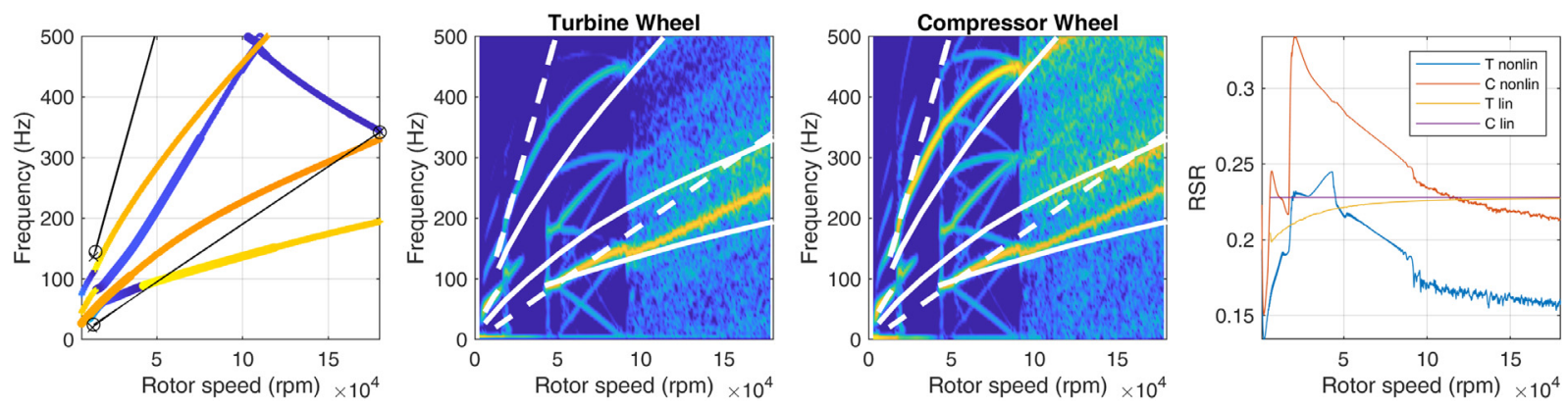

Fig. 14. Comparison of the linearized model $\mathrm{J}$ with run-up simulation including nonlinear forces considering outer clearance $c_{0}=42 \mu \mathrm{m}$. Spectrograms depict vibrations of both impellers as a function of the rotor speed, solid white lines correspond to the unstable modes from the Campbell diagram and dashed lines depict the whirl frequency curves. Comparison of the RSRs resulting from the run-up simulation and the linearized model are depicted on the right.

- The frequency component sub2 is a theoretical whirl frequency of the unstable bearing and cannot be associated with any unstable mode in the Campbell diagram. The frequency of component sub2 differs noticeably from the theoretical whirl frequency with increasing distance from the second bifurcation. With higher outer clearance, the difference is increasing more rapidly. These differences can be observed because the speed of the floating ring calculated during the run-up varies from the speed calculated analytically, which results in varying whirl frequencies.

- The simulated whirl frequency might cross the frequency of the unstable cylindrical mode that is depicted in Fig. 14. At this point, the whirl frequency becomes locked to the frequency of the unstable mode, which leads to oil whip in the inner film. The oil whip in the inner film vanishes if the inner film becomes unstable and the conditions for the total instability are not met.

- The bifurcation at which component sub3 appears corresponds to the predicted speed at which the forward conical mode becomes unstable. Interestingly, the motion associated with sub3 is performed at the theoretical whirl frequency 
rather than at the frequency of the unstable conical mode. If the oil whip condition described in the previous item is reached, the frequency of motion sub3 jumps closer to the frequency of the unstable conical mode.

- In some cases, a new component whose frequency is higher than that of sub3 appears at very high rotor speeds. This new component will appear if the theoretical whirl frequency crosses the frequency of the unstable backward conical mode, see Fig. 14.

\section{Conclusion}

This work has provided a coherent theory for the linear analysis of the FRBs that are used most often in turbocharger applications. Although the turbocharger rotor supported on the FRBs is a nonlinear system, the linear analysis can provide fundamental insight into the turbocharger rotordynamics efficiently. It is because the linearization process and modal analysis is a far less demanding problem than the simulation of a run-up and subsequent data post-processing.

It has been demonstrated that some linear methods can be used in order to predict bifurcations in the rotor response. In particular, the bifurcations that lead to the excitation of gyroscopic conical deflection shapes (components sub1 and sub3) can be associated with the loss of the stability of particular modes. Interestingly, the frequency of the resulting motions is not necessarily equal to the frequency of the unstable mode shape. If a theoretical frequency of motion in the unstable bearing (whirl frequency) is lower than the frequency of the unstable mode, the resulting motion is performed at this lower frequency.

Rules determining behaviour of component sub2 (gyroscopic cylindrical deflection shape) are more complicated. We assume that sub2 can appear after the mode associated with sub1 becomes stable. Furthermore, there should be an unstable cylindrical mode or fluid-induced instability in the inner film in both bearings. The motion associated with component sub2 then appears at the theoretical whirl frequency of the inner film. We have predicted the frequency of sub2 correctly only in a short range after its onset. When the rotor speed increased enough, a noticeable difference between the theoretical whirl frequency and the actual frequency of sub2 occurred. As expected, once the frequency of sub2 crosses the aforementioned frequency of the unstable cylindrical mode, it becomes locked into it, which leads to oil whip. The locking can cause jumping of sub3. So far this behaviour has been explained as a jump from sub1 to sub3, see Section 1 and Fig. 2(f)-(h). The presented study suggests that at least some of these jumps involve only sub2 and sub3 and occur due to oil whip in the outer film.

In order to provide results in such detail, several methods of linear representation or linear approximation of the fluid film forces in the FRB have been introduced. The methods span from the simplest isotropic approximation of the whole FRB to the most complex one which includes separated linearization in the inner and the outer fluid film, the rotational degree of freedom for the floating rings and the independently determined ring speed. The analysis of the resulting composite Campbell diagrams, which show not only values of eigenfrequencies but also indicate damping and precession, has been performed. It has been demonstrated that methods A-D which neglect motion of the floating rings have the low capability in the prediction of the conical and the cylindrical modes because their topology does not correspond well to the physical reality.

Methods F-J with a more precise linearized description of the FRB have been introduced. The aim was to obtain the Campbell diagrams that correspond better to the actual (nonlinear) behaviour of the turbocharger. However, this goal has not been fully achieved because there are only slight differences in modal properties predicted by methods F-J. The more precise description of the FRBs (separated linearization in both fluid films, the RSR determination or degree of freedom for the floating ring rotation) does not lead to more accurate prediction of the modal properties. Computational demands of the introduced methods have been studied. Method J, which is, from the physical point of view, the most precise in the description of FRBs, is considerably more demanding than other three methods. It means that methods F, G, and I are more appropriate for the analysis of the modal properties than method $\mathrm{J}$.

\section{Declaration of Competing Interest}

The authors declare that they have no known competing financial interests or personal relationships that could have appeared to influence the work reported in this paper.

\section{Acknowledgments}

This publication was supported by the Motivation system of the University of West Bohemia - Part POSTDOC, by the project No. 17-15915S of the Czech Science Foundation which is part of project LO1506 of the Czech Ministry of Education, Youth and Sports, and by the project SGS-2019-009.

\section{Appendix}


Table 2

Parameters of the typical turbocharger, adopted from [10].

\begin{tabular}{|c|c|c|c|c|c|}
\hline $\begin{array}{l}\text { Shaft parameters } \\
\text { Finite element number }\end{array}$ & Length $[\mathrm{mm}]$ & Outer diameter $[\mathrm{mm}]$ & Inner diameter $[\mathrm{mm}]$ & Density $\left[\mathrm{kg} \cdot \mathrm{m}^{-3}\right]$ & Young's modulus [MPa] \\
\hline 1 & 33 & 11 & 0 & 7860 & $2 \cdot 10^{5}$ \\
\hline 2 & 39 & 11 & 0 & 7860 & $2 \cdot 10^{5}$ \\
\hline 3 & 33 & 11 & 0 & 7860 & $2 \cdot 10^{5}$ \\
\hline \multicolumn{6}{|l|}{ Bearings parameters } \\
\hline & \multicolumn{2}{|l|}{ Turbine side } & \multicolumn{2}{|l|}{ Compressor side } & \\
\hline & Inner film & Outer film & Inner film & Outer film & \\
\hline dynamic viscosity [Pa.s] & $4.9 \cdot 10^{-3}$ & $4.9 \cdot 10^{-3}$ & $6.4 \cdot 10^{-3}$ & $6.4 \cdot 10^{-3}$ & \\
\hline clearance $[\mu \mathrm{m}]$ & 34 & $\langle 36 ; 46\rangle$ & 34 & $\langle 36 ; 46\rangle$ & \\
\hline bearing length $[\mathrm{mm}]$ & 6.5 & 9 & 6.5 & 9 & \\
\hline bearing radius $[\mathrm{mm}]$ & 5.5 & 8 & 5.5 & 8 & \\
\hline FR mass $[\mathrm{kg}]$ & 7.2 & & 7.2 & & \\
\hline FR moment of inertia $\left[\mathrm{kg} \cdot \mathrm{m}^{2}\right]$ & $33.641 \cdot 10^{-8}$ & & $33.641 \cdot 10^{-8}$ & & \\
\hline \multicolumn{6}{|l|}{ Wheels parameters } \\
\hline & \multicolumn{2}{|l|}{ Turbine side } & \multicolumn{3}{|l|}{ Compressor side } \\
\hline mass $[\mathrm{kg}]$ & \multirow{3}{*}{\multicolumn{2}{|c|}{$\begin{array}{l}0.326 \\
8.1 \cdot 10^{-5} \\
7.7 \cdot 10^{-5}\end{array}$}} & \\
\hline polar moment of inertia $\left[\mathrm{kg} \cdot \mathrm{m}^{2}\right]$ & & & $4.4 \cdot 10^{-5}$ & & \\
\hline moment of inertia $\left[\mathrm{kg} \cdot \mathrm{m}^{2}\right]$ & & & $3.27 \cdot 10^{-5}$ & & \\
\hline
\end{tabular}

\section{References}

[1] H. Nguyen-Schäfer, Rotordynamics of Automotive Turbochargers, Springer-Verlag, Berlin, 2012.

[2] B. Schweizer, Oil whirl, oil whip and whirl/whip synchronization occurring in rotor systems with full-floating ring bearings, Nonlinear Dyn. 57 (4) (2009) 509-532, doi:10.1007/s11071-009-9466-3.

[3] A. Muszynska, Whirl and whip - rotor/bearing stability problems, J. Sound Vib. 110 (3) (1986) 443-462, doi:10.1016/S0022-460X(86)80146-8.

[4] L.S. Andres, J. Kerth, Thermal effects on the performance of floating ring bearings for turbochargers, Proc. Inst. Mech. Eng. J: J. Eng. Tribol. 218 (5) (2004) 437-450, doi:10.1243/1350650042128067.

[5] J.K. Wang, M.M. Khonsari, Bifurcation analysis of a flexible rotor supported by two fluid-film journal bearings, J. Tribol. 128 (3) (2006) 594-603, doi:10.1115/1.2197842.

[6] L.S. Andres, J.C. Rivadeneira, K. Gjika, C. Groves, G. LaRue, Rotordynamics of small turbochargers supported on floating ring bearings-highlights in bearing analysis and experimental validation, J. Tribol. 129 (2) (2007) 391-397, doi:10.1115/1.2464134.

[7] R. Holmes, M.J. Brennan, B. Gottrand, Vibration of an automotive turbocharger - a case study, in: Proceedings of the Eighth International Conference on Vibrations in Rotating Machinery, Institution of Mechanical Engineers, Bury St. Edmunds, 2004.

[8] B. Schweizer, Total instability of turbocharger rotors - physical explanation of the dynamic failure of rotors with full-floating ring bearings, J. Sound Vib. 328 (1-2) (2009) 156-190, doi:10.1016/j.jsv.2009.03.028.

[9] B. Schweizer, Dynamics and stability of turbocharger rotors, Arch. Appl. Mech. 80 (9) (2010) 1017-1043, doi:10.1007/s00419-009-0331-0.

[10] L. Tian, W. Wang, Z. Peng, Effects of bearing outer clearance on the dynamic behaviours of the full floating ring bearing supported turbocharger rotor Mech. Syst. Signal Process. 31 (2012) 155-175, doi:10.1016/j.ymssp.2012.03.017.

[11] E. Woschke, C. Daniel, S. Nitzschke, Excitation mechanisms of non-linear rotor systems with floating ring bearings - simulation and validation, Int. J. Mech. Sci. 134 (December) (2017) 15-27, doi:10.1016/j.ijmecsci.2017.09.038.

[12] C. Zhang, R. Men, H. He, W. Chen, Effects of circumferential and axial grooves on the nonlinear oscillations of the full floating ring bearing supported turbocharger rotor, Proc. Inst. Mech. Eng. J: J. Eng. Tribol. 233 (5) (2019) 741-757, doi:10.1177/1350650118800581.

[13] L. Tian, W. Wang, Z. Peng, Nonlinear effects of unbalance in the rotor-floating ring bearing system of turbochargers, Mech. Syst. Signal Process. 34 (1-2) (2013) 298-320, doi:10.1016/j.ymssp.2012.07.017.

[14] A.D. Shaw, A.R. Champneys, M.I. Friswell, Normal form analysis of bouncing cycles in isotropic rotor stator contact problems, Int. J. Mech. Sci. 155 (May) (2019) 83-97, doi:10.1016/j.ijmecsci.2019.02.035.

[15] L. Smolík, M. Hajžman, M. Byrtus, Investigation of bearing clearance effects in dynamics of turbochargers, Int. J. Mech. Sci. 127 (July) (2017) 62-72, doi:10.1016/j.ijmecsci.2016.07.013.

[16] H. Roy, S. Chandraker, Dynamic study of viscoelastic rotor: modal analysis of higher order model considering various asymmetries, Mech. Mach. Theory 109 (2017) 65-77, doi:10.1016/j.mechmachtheory.2016.11.003.

[17] C.-Y. Joh, C.-W. Lee, Use of dFRFs for diagnosis of asymmetric/anisotropic properties in rotor-bearing system, J. Vib. Acoust. 118 (1) (1996) 64-69, doi:10.1115/1.2889636.

[18] C.L. Kessler, Complex modal analysis of rotating machinery, University of Cincinnati, 1999 Ph.D. thesis.

[19] M. Chouksey, J. Dutt, S. Modak, Modal analysis of rotor-shaft system under the influence of rotor-shaft material damping and fluid film forces, Mech. Mach. Theory 48 (2012) 81-93, doi:10.1016/j.mechmachtheory.2011.09.001.

[20] J.W. Lund, Review of the concept of dynamic coefficients for fluid film journal bearings, J. Tribol. 109 (1) (1987) 37-41, doi:10.1115/1.3261324.

[21] J. Vance, F. Zeidan, B. Murphy, Machinery Vibration and Rotordynamics, John Wiley \& Sons, Ltd, 2010.

[22] O. Reynolds, On the theory of lubrication and its application to Mr. Beauchamp tower's experiments, including an experimental determination of the viscosity of olive oil, Philos. Trans. R. Soc. Lond. 177 (1886) 157-234, doi:10.1098/rstl.1886.0005.

[23] D. Sfyris, A. Chasalevris, An exact analytical solution of the Reynolds equation for the finite journal bearing lubrication, Tribol. Int. 55 (2012) $46-58$.

[24] Y. Bastani, M. de Queiroz, A new analytic approximation for the hydrodynamic forces in finite-length journal bearings, J. Tribol. 132 (1) (2009) 01450201-014502-9, doi:10.1115/1.4000389.

[25] C.E. Merelli, D.O. Baril, G.G. Vignolo, L.M. Quinzani, Dynamic coefficients of finite length journal bearing. evaluation using a regular perturbation method, Int. J. Mech. Sci. 151 (2019) 251-262, doi:10.1016/j.ijmecsci.2018.11.018,

[26] Š. Dyk, J. Rendl, M. Byrtus, L. Smolk, Dynamic coefficients and stability analysis of finite-length journal bearings considering approximate analytical solutions of the Reynolds equation, Tribol. Int. 130 (2019) 229-244, doi:10.1016/j.triboint.2018.09.011.

[27] Y. Hori, Hydrodynamic Lubrication, Springer-Verlag, Tokyo, 2006. 
[28] S. Nitzschke, E. Woschke, C. Daniel, Application of regularised cavitation algorithm for transient analysis of rotors supported in floating ring bearings, in: K.L. Cavalca, H.I. Weber (Eds.), Proceedings of the Tenth International Conference on Rotor Dynamics-IFToMM, Springer International Publishing, Cham, 2019, pp. 371-387.

[29] D. Cheng, X. Hu, T. Shen, Linearization of Nonlinear Systems, Springer Berlin Heidelberg, Berlin, Heidelberg, pp. 279-313. 10.1007/978-3-642-11550$9+10$

[30] C.-T. Chen, Linear System Theory and Design, 3rd ed., Oxford University Press, Inc., New York, NY, USA, 1998.

[31] D. Childs, Turbomachinery Rotordynamics: Phenomena, Modeling, and Analysis, Wiley-Interscience publication, Wiley, 1993.

[32] L. Tian, Investigation into nonlinear dynamics of rotor-floating ring bearing systems in automotive turbochargers, University of Sussex, 2012 Ph.D. thesis.

[33] L. Smolík, J. Rendl, Š. Dyk, P. Polach, M. Hajžman, Threshold stability curves for a nonlinear rotor-bearing system, J. Sound Vib. 442 (2019) 698-713, doi:10.1016/j.jsv.2018.10.042. 